\title{
Cross Section Measurements at LANSCE for Defense, Science and Applications
}

\author{
Ronald O. Nelson ${ }^{a}$ \\ M.S. H855, Los Alamos Neutron Science Center (LANSCE), Los Alamos, NM 87545, USA
}

\begin{abstract}
The Los Alamos Neutron Science Center (LANSCE) has three neutron sources that are used for nuclear science measurements. These sources are driven by an $800 \mathrm{MeV}$ proton linear accelerator and cover an energy range from sub-thermal to hundreds of MeV. Research at the facilities is performed under the auspices of a US DOE user program under which research proposals are rated for merit by a program advisory committee and are scheduled based on merit and availability of beam time. A wide variety of instruments is operated at the neutron flight paths at LANSCE including neutron detector arrays, gamma-ray detector arrays, fission fragment detectors, and charged particle detectors. These instruments provide nuclear data for multiple uses that range from increasing knowledge in fundamental science to satisfying data needs for diverse applications such as nuclear energy, global security, and industrial applications. Highlights of recent research related to cross sections measurements are presented, and future research initiatives are discussed.
\end{abstract}

\section{Introduction}

Los Alamos Neutron Science Center (LANSCE) provides neutron beams and nuclear science instruments for a diverse program of measurements. Many of these experiments provide cross section data for nuclear processes. Working with nuclear physics theorists and nuclear data evaluators, LANSCE staff and collaborators work to provide high quality data evaluations for applications and to add to the understanding of nuclear reactions and nuclear structure, as well as other physical processes and phenomena.

A brief description of the facilities and user program is given, followed by highlights of current research in relation to cross section measurements. Future directions are outlined in the conclusion.

\section{Facilities}

The LANSCE accelerator and neutron production targets are described in [1]. The $800 \mathrm{MeV}$ pulsed proton linear accelerator provides beams to multiple areas enabling a very wide range of activities to be carried out simultaneously. For lower energy neutrons a moderated tungsten spallation target is operated with a typical beam current of 100 micro amperes and a repetition rate of 20 $\mathrm{Hz}$ at the Lujan Neutron Scattering Center. Both water and liquid hydrogen moderators are available with 16 flight paths ranging from less than 8 meters to over 60 meters. The useful energy range for the moderated source is from sub-thermal to about $1 \mathrm{MeV}$. For higher energy neutrons a bare tungsten target is used at the WNR facility. Typical beam currents range from 2 to 5 micro amperes, with typical pulse spacings of 1.8 microseconds. The six flight paths available range from about 10 meters to 90 meters. Recently one of the high-power radio frequency drivers of the accelerator has been upgraded to allow operation at $120 \mathrm{~Hz}$ rather than $60 \mathrm{~Hz}$ that has been the limit for some years. Three other RF drivers will be upgraded in the coming years for reliable operation.

A very high effective neutron flux is provided in the $\mathrm{eV}$ to $100 \mathrm{keV}$ energy range by a Lead Slowing Down Spectrometer (LSDS) that is operated in the "Blue Room" where the $800 \mathrm{MeV}$ proton beam is available for experiments. The LSDS consists of a cube of pure $\mathrm{Pb}$ with a side length of 1.2 meters. The LSDS is operated in collaboration with the CEA, Bruyeres-le-Chatel, France. Experiments with extremely small $(10 \mathrm{ng})$ and radioactive samples are performed with the LSDS.

\section{The Nuclear Science User Program}

The LANSCE Nuclear Science User Program accepts proposals in three main subgroups, (1) basic science, (2) nuclear technology including industry users, and (3) defense science. For the four month accelerator operating period in 2013, there were 259 users who made a total of 558 separate visits to conduct experiments at LANSCE in Nuclear Science. The users were approximately equally divided among university, industry and national laboratory staff. Users from non-U.S. institutions are an important part of the research efforts.

\footnotetext{
${ }^{a}$ Corresponding author: rnelson@lanl.gov
} 


\section{Cross Section Measurements}

Some of the main instruments that are currently providing nuclear data at LANSCE are described below.

\subsection{GEANIE - gamma-ray cross sections}

The Germanium Array for Neutron Induced-Excitations (GEANIE) consists of 20 high-purity germanium detectors with BGO escape-suppression shields [2]. Up to ten of the detectors are planar Ge detectors with Be windows that are useful for measuring low-energy gamma rays and $\mathrm{x}$ rays. The other detectors are coaxial geometry and typically are used in the energy range from approximately $70 \mathrm{keV}$ to several $\mathrm{MeV}$. GEANIE is located on a 20 -meter flight path at the WNR high-energy neutron source. The time-of-flight technique is used to make measurements in the energy range from hundreds of $\mathrm{keV}$ to hundreds of $\mathrm{MeV}$ simultaneously. The GEANIE array is shown in Fig. 1.

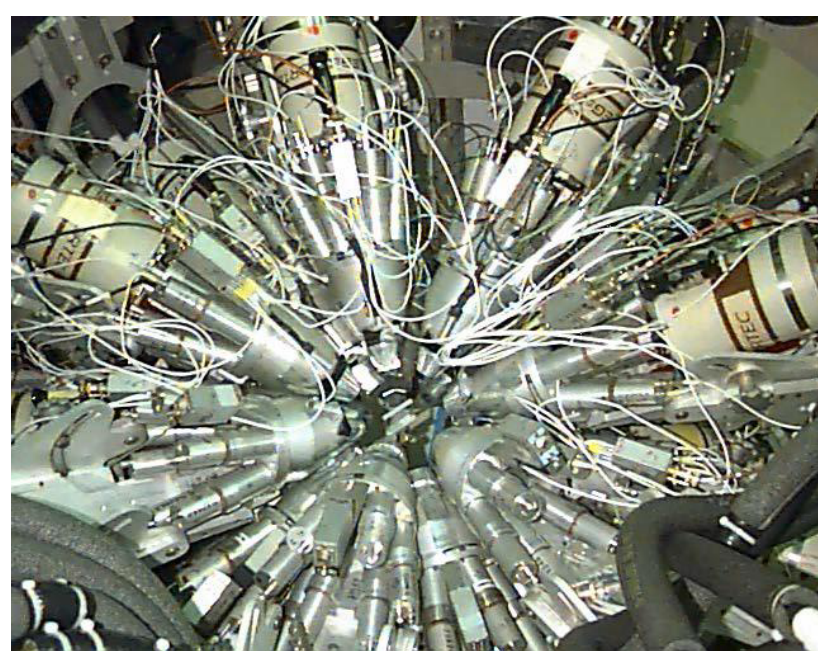

Figure 1 Top view of the GEANIE array of germanium detectors with the sample at the center.

Recent efforts have focused on developing reference cross sections to enable more accurate relative measurements. LiF and Ti have been identified as good potential candidates. The isotropic $478-\mathrm{keV}$ gamma ray from ${ }^{7} \mathrm{Li}\left(\mathrm{n}, \mathrm{n}^{\prime}\right.$ gamma) for $1<\mathrm{En}<8 \mathrm{MeV}$ and the 983$\mathrm{keV}$ gamma ray from ${ }^{48} \mathrm{Ti}\left(\mathrm{n}, \mathrm{n}\right.$ 'gamma) for $4<\mathrm{E}_{\mathrm{n}}<15$ $\mathrm{MeV}$ are the two main gamma rays selected. LiF optical windows provide a material with excellent properties for the lower energy case, and high-purity Ti foils are well- suited for the higher energy region. Preliminary and previous data for Li(n,n'gamma) are shown in Fig. 2.

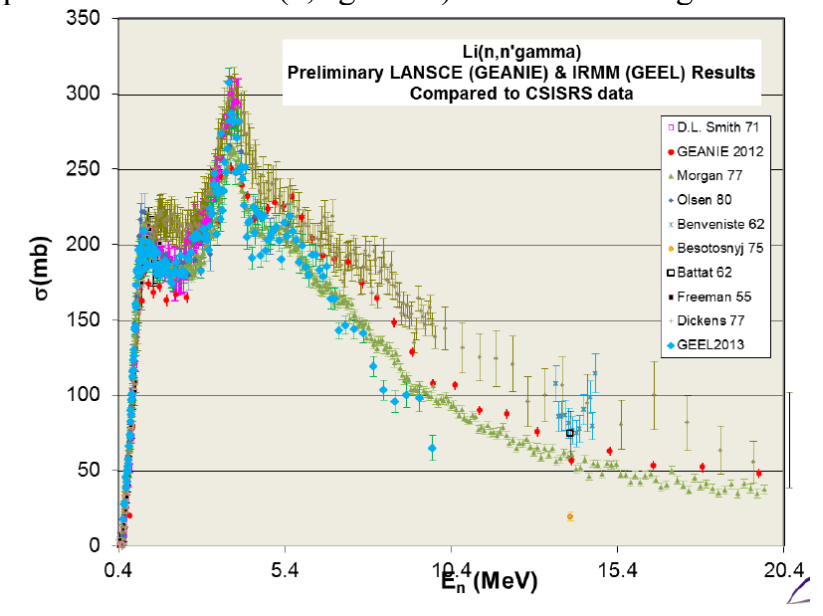

Figure 2 Preliminary results of $\mathrm{Li}(\mathrm{n}, \mathrm{n}$ 'gamma) measurements from GEANIE and from GEEL plotted with data from previous measurements. Differences between the recent data sets remain to be resolved.

\subsection{Chi-Nu - fission neutron spectra}

The Chi-Nu project is an intense effort to make accurate measurements of the neutron spectrum following neutron-induced fission of ${ }^{239} \mathrm{Pu}$ and ${ }^{235} \mathrm{U}$ that extend as low as $\mathrm{E}_{\mathrm{n}}=50 \mathrm{keV}$ and as high in outgoing neutron energy as $12 \mathrm{MeV}$, in the $\mathrm{MeV}$ range of incident neutron energies.[3] For these measurements an array of ${ }^{6} \mathrm{Li}$-glass detectors has been designed and constructed for the lowenergy measurements, and an array of liquid scintillator detectors has been designed and fabricated for the higher energy measurements. A drawing of the higher-energy array is shown in Fig. 3.

These experiments make use of very detailed and extensive Monte Carlo modelling to understand and to help reduce sources of background neutrons that make accurate measurements extremely difficult in the outgoing neutron low-energy region.

Following the measurements of fission neutron spectra, these arrays of neutron detectors can be employed for detailed studies of inelastic scattering in which, by tagging on specific inelastic gamma rays, the excitation energy of the nucleus and the energies and angular distributions of the neutrons can be determined. This technique has been demonstrated by the FIGARO collaboration at LANSCE. 


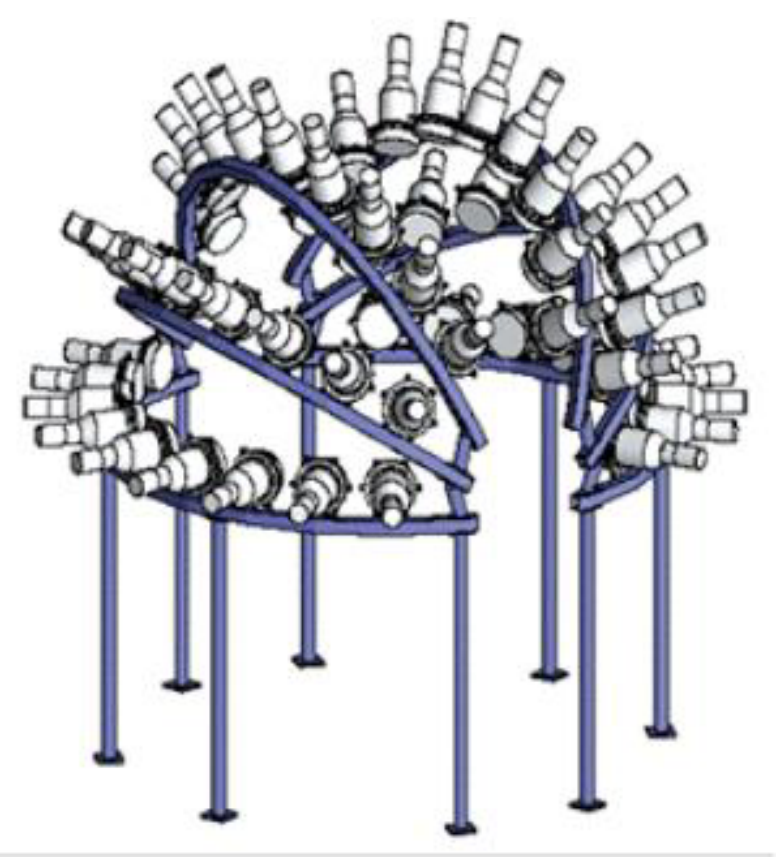

Figure 3 The Chi-Nu array of high-energy neutron detectors consisting of 54 liquid scintillator detectors with a 1 meter flight path from the fission detector at the center of the array.

\subsection{TPC - neutron-induced fission cross sections}

The Neutron-Induced Fission-Fragment Tracking Experiment (NIFFTE) is using a specially designed and fabricated Time Projection Chamber (TPC) to measure neutron-induced fission cross sections in a new way with the goal of better than $1 \%$ accuracy in the absolute value.[4] The experiment collaboration includes Lawrence Livermore National Laboratory (TPC and readout design and fabrication), Los Alamos National Laboratory (neutron beam delivery and TPC operation), and university collaborators from a number of schools who have responsibility for important elements of the effort.

The TPC uses a collection foil with a pixelated collection plane and time tracking that allows 3-D charged particle track reconstruction. Particle identification is achieved based on the ionization tracks with alpha particles clearly differentiated from fission fragments. Measurements are being made relative to ${ }^{235} \mathrm{U}$ and $n-p$ scattering standard cross sections. The TPC is shown installed on the 90 degree left flight path at WNR in Fig. 4.

\subsection{Neutron Capture from Total Cross Sections}

A recent Los Alamos Report [5] describes a method of using total neutron cross section measurements to infer neutron capture cross sections for unstable nuclei that cannot be measured by standard neutron capture techniques, even with the best existing detectors and neutron sources.

The method relies on neutron total cross section measurements that can be made using very small (10 microgram, $0.5 \mathrm{~mm}$ diameter) samples of radioactive materials in a conventional total cross section geometry.
Values of average resonance spacings, D0, and neutron strength functions, S0, are determined from the total cross section measurements, and then the Nuclear Statistical Model is used to calculate the capture cross section. This has been demonstrated for the case of ${ }^{151} \mathrm{Sm}(\mathrm{n}$, gamma) with excellent agreement between the cross section inferred from previous total cross section data and actual measurements of the capture cross section.

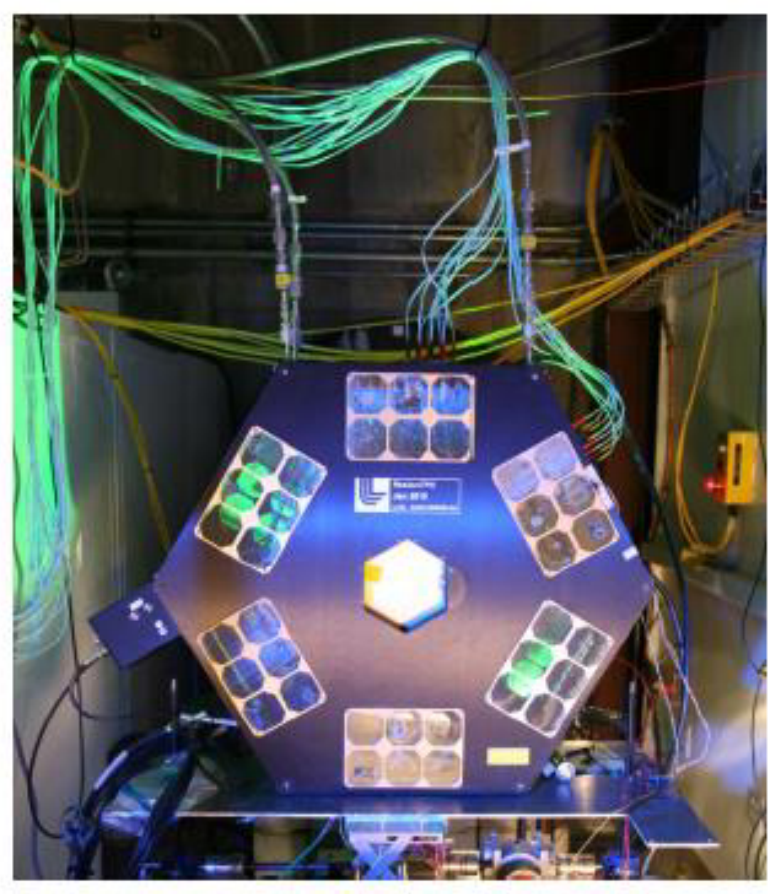

Figure 4 The TPC and associated 6000 channel readout system are shown in the 90 degree left flight path at the WNR facility.

Samples that are good candidates for this technique include: ${ }^{88} \mathrm{Y},{ }^{107} \mathrm{Pd},{ }^{110 \mathrm{~m}} \mathrm{Ag},{ }^{134} \mathrm{Cs},{ }^{147} \mathrm{Nd},{ }^{147} \mathrm{Pm},{ }^{152} \mathrm{Eu}$, ${ }^{154} \mathrm{Eu},{ }^{155} \mathrm{Eu},{ }^{153} \mathrm{Gd},{ }^{160} \mathrm{~Tb},{ }^{161} \mathrm{~Tb},{ }^{163} \mathrm{Ho},{ }^{166} \mathrm{Ho},{ }^{169} \mathrm{Er}$, ${ }^{170} \mathrm{Tm},{ }^{171} \mathrm{Tm},{ }^{175} \mathrm{Yb},{ }^{179} \mathrm{Ta},{ }^{185} \mathrm{~W},{ }^{186} \mathrm{Re},{ }^{191} \mathrm{Os},{ }^{192} \mathrm{Ir},{ }^{193} \mathrm{Pt}$, ${ }^{198} \mathrm{Au}$. All of these have interest for astrophysics or defense programs.

\section{Conclusion}

A wide variety of cross section measurements for neutron-induced reactions are performed at LANSCE: fast-neutron induced gamma-ray measurements, fission measurements of outgoing neutron spectra as well as precision fission cross sections and photons emission. Low-energy neutron capture and fission measurements at LANSCE using the DANCE gamma-ray detector are described in other contributions to these proceedings.

Operation of the accelerator at full $120 \mathrm{~Hz}$ duty cycle starts in October 2014 with all LANSCE neutron production targets in operation. The LANSCE Nuclear Science User Program had a record number of users in 2013 and continues in 2014.

New initiatives are planned to expand capabilities for elastic and inelastic neutron scattering measurements, and for total cross sections on small and radioactive samples. 


\section{Acknowledgements}

The author would like to thank all of the co-workers and collaborators whose work is represented. LANSCE is supported by U. S. DOE under contract \#DE-AC5206NA25396.

\section{References}

1. P.W. Lisowski and K.F. Schoenberg, Nucl. Instrum. Methods Phys. Res. A, 562, 910 (2005).

2. J. A. Becker and R.O. Nelson, "New Opportunities in Nuclear Science with GEANIE at LANSCE", Nuclear Physics News 7, 11 (1997) ; R.O. Nelson, et al., «GEANIE at WNR/LANSCE -- A new instrument for neutron science,» International conference on nuclear data for science and technology; Trieste (Italy); 20-24 May 1997; Los Alamos National Laboratory Report LA-UR-97-1824 (1997).

3. R.C. Haight et al. «The LANL/LLNL Prompt Fission Neutron Spectrum Program at LANSCE and approach to uncertainties, « Proceedings of International Workshop on Nuclear Data Covariances, Santa Fe, NM April 28- May 1, 2014 Atomic and Nuclear Data Sheets (to be published), Los Alamos National Labortory Report LA-UR-1424648 (2014).

4. M. Heffner, et al., Nucl. Instrum. Meth. Phys. Res. A 759, 50 (2014).

5. P. E. Koehler, Los Alamos National Laboratory Report LA-UR-14-21466 (2014). 